\title{
Abundances of seven red giants in the open cluster NGC $3114 \dagger$
}

\author{
C. B. Pereira and C. Quireza \\ Observatório Nacional, Rua General José Cristino 77, 20921-400 São Cristóvão, \\ Rio de Janeiro, RJ Brazil \\ email: [claudio, quireza] @on.br
}

\begin{abstract}
We present a chemical analysis of seven red giants in the open cluster NGC 3114. Our main goal is to investigate the chemical composition of this cluster, which is not yet available in the literature. We employed the FEROS spectrograph on the ESO $2.2 \mathrm{~m}$ telescope. Atmospheric parameters and metallicity were derived from the measured equivalent widths of several iron lines using the spectral code MOOG and Kurucz model atmospheres. We obtained the abundances of $\mathrm{O}, \mathrm{Al}, \mathrm{Ca}, \mathrm{Mg}, \mathrm{Si}, \mathrm{Ti}, \mathrm{Ni}, \mathrm{Cr}, \mathrm{Sc}, \mathrm{Y}, \mathrm{Zr}, \mathrm{La}, \mathrm{Ce}$ and $\mathrm{Nd}$ by measuring the equivalent widths of the absorption lines of these elements. A mean metallicity of $[\mathrm{Fe} / \mathrm{H}]=0.05 \pm 0.13$ relative to the Sun was determined from the data of the red-giant members. This result is in good agreement with the Galactic-disk radial distribution of iron traced by open clusters. We did not find any intrinsic star-to-star scatter in the [element/Fe] ratios for the stars in this cluster. We compare our results with investigations of other open clusters. An age of $8.2 \mathrm{Gyr}$ is derived from isochrone fits.
\end{abstract}

Keywords. open clusters and associations: individual (NGC 3114), Galaxy: abundances

\section{Introduction}

Open clusters probe the structure and evolution of the Galactic disk. They are tools for the investigation of properties like age, interstellar dust and metallicity distribution, the age-metallicity relation, as well as the dynamics of the disk. They are also good targets for studies of stellar evolution. Relative to field stars, star clusters should allow more accurate evaluations of their age, metallicity, distance and velocity, since such properties should be common for all of their members.

In this paper, we present spectroscopic observations of seven red giants in the open cluster NGC 3114. This cluster is located in the Carina spiral feature, a region of heavy contamination by field stars (Carraro \& Patat 2001). There are some photometric studies for NGC 3114 (Jankowitz \& McCosh 1963; Schneider \& Weiss 1988; Clariá et al. 1989; Sagar \& Sharpless 1991; Carraro \& Patat 2001; Paunzen et al. 2003), but high-resolution data dedicated to abundance studies are not available in the literature. Thanks to the radial-velocity surveys of González \& Lapasset (2001) and, more recently, Mermilliod et al. (2008), several stars have been identified as members of NGC 3114, including the seven red giants analyzed here. Our aim is to provide accurate abundances for stars in this cluster.

Spectral-line observations are summarized in Section 2. In Section 3, we describe our analysis of the data and the main results. In Section 4, we discuss the astrophysical implications of our results. A brief conclusion follows in Section 5 .

$\dagger$ Based on observations with the $2.2 \mathrm{~m}$ telescope at the European Southern Observatory (La Silla, Chile). 
Table 1. Log of the observations and relevant information of the target stars.

\begin{tabular}{|c|c|c|c|c|c|c|c|}
\hline $\mathrm{ID}^{a}$ & H D & $\begin{array}{c}V^{a} \\
(\mathrm{mag})\end{array}$ & $(B-V)^{a}$ & $\begin{array}{c}\mathrm{RV}^{a} \\
\mathrm{~km} \mathrm{~s}^{-1}\end{array}$ & $\begin{array}{c}\mathrm{RV}^{b} \\
\mathrm{~km} \mathrm{~s}^{-1}\end{array}$ & Date obs & $\begin{array}{c}\operatorname{Exp} \\
(\mathrm{s})\end{array}$ \\
\hline 6 & 87109 & 7.6 & 1.29 & $-1.43 \pm 0.23$ & $-1.31 \pm 0.49$ & 2008 Apr 9 & 600 \\
\hline 150 & 87479 & 7.9 & 1.17 & $-2.19 \pm 0.51$ & $-1.71 \pm 0.61$ & 2008 Apr 9 & 420 \\
\hline 170 & 87526 & 7.3 & 0.89 & $-1.95 \pm 0.40$ & $-2.38 \pm 0.17$ & 2008 Apr 9 & 600 \\
\hline 181 & 87566 & 8.3 & 1.28 & $-2.18 \pm 0.10$ & $-2.27 \pm 0.23$ & $2008 \mathrm{Apr} 10$ & 420 \\
\hline 238 & 304859 & 8.5 & 1.27 & $-1.72 \pm 0.18$ & $-1.40 \pm 0.34$ & 2008 Dec 22 & 600 \\
\hline 262 & 87833 & 8.6 & 1.16 & $-1.20 \pm 0.23$ & $-1.22 \pm 0.17$ & $2008 \mathrm{Apr} 10$ & 600 \\
\hline 283 & 304864 & 7.7 & 1.25 & $-1.73 \pm 0.31$ & $-1.41 \pm 0.36$ & 2008 Dec 22 & 600 \\
\hline
\end{tabular}

${ }^{a}$ Mermilliod et al. $(2008) ;{ }^{b}$ this work.

\section{Observational data}

\subsection{Properties of $N G C 3114$}

NGC 3114 (C 1001-598, Cl VDBH 86; $\alpha=10^{\mathrm{h}} 02^{\mathrm{m}} .0, \delta=-60^{\circ} 06^{\prime}(\mathrm{J} 2000.0) ; l=283^{\circ}$, $\left.b=-04^{\circ}\right)$, is a young open cluster projected onto the outskirts of the Carina complex (Feinstein 1995). Carraro \& Patat (2001) presented a CCD UBVRI photometric study of the cluster and obtained magnitudes down to $V \simeq 22.0 \mathrm{mag}$. Their results for the age, heliocentric distance and reddening were, respectively, $3.0 \times 10^{8}$ years, $920 \pm 50 \mathrm{pc}$ and $E(B-V)=0.07 \pm 0.03 \mathrm{mag}$. Similar values were also found by González \& Lapasset (2001), who derived an age of $1.6 \times 10^{8}$ years, a distance modulus $\left(V-M_{V}\right)=9.8 \pm 0.2$ mag and a reddening of $E(B-V)=0.07 \pm 0.01$ mag.

Our sample includes seven red giants originally selected from the radial-velocity catalog of Mermilliod et al. (2008). For the seven stars in our sample, González \& Lapasset (2001) computed membership probabilities greater than the $95 \%$ level.

\subsection{Observations}

We obtained high-resolution spectra for these seven stellar members of NGC 3114 using the ESO 2.2m telescope (La Silla, Chile) with the FEROS echelle spectrograph (Kaufer et al. 1999). The wavelength coverage is from 4000 to $9200 \AA$. Table 1 lists the observation $\log$ and additional information about the observed stars. The stars in our sample closely share the same radial velocity (RV). Our values are close to the results of Mermillod et al. (2008), who provided $\langle\mathrm{RV}\rangle=-1.72 \mathrm{~km} \mathrm{~s}^{-1}$. We note, however, that González \& Lapasset (2001) estimated values that are systematically lower than ours, by a factor of about two $\left(\langle\mathrm{RV}\rangle=-3.8 \pm 0.3 \mathrm{~km} \mathrm{~s}^{-1}\right)$.

\section{Analysis and results}

The spectra of the red giants in our sample show many atomic absorption lines of Fe I and Fe II as well as transitions due to CaI, Na I, Mg I, Al I, Sc II, Ti I, Vi, Cr I, Co I, Ni I, Zn I, Cu I, Y II, Ba II, La II, Ce II, Nd II and Eu II. We have chosen a set of lines sufficiently unblended to yield reliable abundances.

The determination of atmospheric parameters, effective temperatures $\left(T_{\text {eff }}\right)$, surface gravities $(\log g)$, microturbulent velocities $(\xi)$ and metallicity $([\mathrm{Fe} / \mathrm{H}]$; throughout, we use the notation $[\mathrm{X} / \mathrm{H}]=\log (N(\mathrm{X}) / N(\mathrm{H}))_{\star}-\log \left(N(\mathrm{X}) / N(\mathrm{H})_{\odot}\right)$ is prerequisite for determination of the final abundance. These parameters were derived from the measured equivalent widths of the iron lines, using the spectral code MOOG and Kurucz model atmospheres. To derive these parameters, we followed the procedures described in previous studies investigating the spectra of barium and symbiotic stars (Pereira 2005; Pereira \& Roig 2009). The final adopted atmospheric parameters of our sample stars are given in Table 2 .

The internal errors associated with our adopted effective temperatures and microturbulent velocities can be determined from the uncertainty in the slope of the Fe I abundance 
Table 2. Adopted atmospheric parameters, Fe I and Fe II metallicities with the corresponding standard deviations and number of lines employed.

\begin{tabular}{lccccc}
\hline Star & $\begin{array}{c}T_{\text {eff }} \\
(\mathrm{K})\end{array}$ & $\begin{array}{c}\log g \\
(\mathrm{dex})\end{array}$ & $\begin{array}{c}\xi \\
\mathrm{km} \mathrm{s}^{-1}\end{array}$ & {$[\mathrm{Fe} \mathrm{I} / \mathrm{H}] \pm \sigma(\#)$} & {$[\mathrm{Fe} \mathrm{II} / \mathrm{H}] \pm \sigma(\#)$} \\
\hline HD 87109 & 4700 & 1.1 & 2.0 & $-0.05 \pm 0.12(47)$ & $-0.04 \pm 0.12(11)$ \\
HD 87479 & 5000 & 1.3 & 1.9 & $+0.33 \pm 0.15(40)$ & $+0.31 \pm 0.15(7)$ \\
HD 87526 & 5300 & 1.5 & 2.9 & $-0.74 \pm 0.10(71)$ & $-0.74 \pm 0.08(13)$ \\
HD 87566 & 4500 & 1.6 & 1.6 & $+0.01 \pm 0.15(54)$ & $+0.01 \pm 0.14(11)$ \\
HD 87833 & 4900 & 2.0 & 1.9 & $-0.05 \pm 0.16(45)$ & $-0.03 \pm 0.15(10)$ \\
HD 304859 & 4500 & 1.6 & 1.6 & $+0.07 \pm 0.18(51)$ & $+0.08 \pm 0.09(10)$ \\
HD 304864 & 4700 & 1.1 & 2.2 & $+0.01 \pm 0.15(34)$ & $+0.01 \pm 0.26(6)$ \\
\hline
\end{tabular}

Table 3. Mean elemental abundances $([\mathrm{X} / \mathrm{Fe}])$, dispersion and the number of lines used for the abundance determination.

\begin{tabular}{|c|c|c|c|c|c|c|c|}
\hline$[\mathrm{X} / \mathrm{Fe}]$ & HD 87109 & HD 87479 & HD 87526 & HD 87566 & HD 87833 & HD 304859 & HD 304864 \\
\hline $\mathrm{O}$ & -0.45 & - & +0.30 & -0.21 & -0.15 & -0.37 & $<-0.31$ \\
\hline $\mathrm{Mg}$ & $+0.18(2)$ & $+0.17(1)$ & $+0.60(2)$ & $+0.17(2)$ & $+0.20 \pm 0.17(3)$ & - & - \\
\hline Al & $-0.03 \pm 0.19(4)$ & $-0.04 \pm 0.20(4)$ & $+0.17 \pm 0.16(3)$ & $-0.16 \pm 0.18(4)$ & $+0.07 \pm 0.17(5)$ & $+0.16 \pm 0.32(4)$ & - \\
\hline $\mathrm{Si}$ & $+0.03 \pm 0.22(9)$ & $-0.08 \pm 0.19(6)$ & $+0.36 \pm 0.17(8)$ & $0.00 \pm 0.21(9)$ & $+0.07 \pm 0.18(6)$ & $+0.31 \pm 0.21(8)$ & $+0.16 \pm 0.21(7)$ \\
\hline $\mathrm{Ca}$ & $+0.04 \pm 0.18(3)$ & $-0.09 \pm 0.17(3)$ & $+0.13 \pm 0.12(8)$ & $0.00 \pm 0.21(3)$ & $-0.10 \pm 0.15(5)$ & $+0.10 \pm 0.25(3)$ & $-0.11(2)$ \\
\hline $\mathrm{Sc}$ & $+0.05 \pm 0.19(4)$ & $-0.10 \pm 0.20(5)$ & $-0.01 \pm 0.15(6)$ & $0.00 \pm 0.25(6)$ & $-0.15 \pm 0.12(4)$ & $+0.1 \pm 0.23(6)$ & $+0.20 \pm 0.23(3)$ \\
\hline $\mathrm{Ti}$ & $-0.08 \pm 0.20(4)$ & $-0.10 \pm 0.07(3)$ & $-0.15 \pm 0.13(4)$ & $-0.27 \pm 0.19(3)$ & $+0.05 \pm 0.19(5)$ & $-0.13(2)$ & $-0.22(2)$ \\
\hline $\mathrm{Cr}$ & $-0.20(2)$ & $+0 . \overline{06}(1)$ & $+0.18 \pm 0.13(4)$ & $-0.05(2)$ & $-0.13(2)$ & $-0.11(2)$ & $-0.20(2)$ \\
\hline $\mathrm{Ni}$ & $+0.03 \pm 0.28(7)$ & $+0.01 \pm 0.19(5)$ & $-0.10 \pm 0.20(9)$ & $-0.07 \pm 0.22(6)$ & $-0.13 \pm 0.17(7)$ & $-0.08 \pm 0.22(6)$ & $-0.15 \pm 0.24 \quad(6)$ \\
\hline $\mathrm{Y}$ & $+0.04 \pm 0.26(3)$ & - & $+0.02 \pm 0.14(4)$ & $-0.02 \pm 0.31(4)$ & $-0.04 \pm 0.08(3)$ & $-0.04 \pm 0.25(4)$ & - \\
\hline $\mathrm{Zr}$ & $+0.09 \pm 0.17(4)$ & $-0.21 \pm 0.28(3)$ & - & $-0.16 \pm 0.18(4)$ & $+0.20 \pm 0.09(4)$ & $-0.06 \pm 0.20(4)$ & $-0.06 \pm 0.08(2)$ \\
\hline $\mathrm{La}$ & $+0.29 \pm 0.22(6)$ & $-0.17 \pm 0.17(4)$ & $+0.02 \pm 0.22(4)$ & $+0.26 \pm 0.23(2)$ & $+0.25 \pm 0.19(5)$ & $+0.25 \pm 0.23(4)$ & $+0.29(2)$ \\
\hline $\mathrm{Ce}$ & $+0.02 \pm 0.26(5)$ & - & $+0.13 \pm 0.27(11)$ & $+0.17 \pm 0.26(5)$ & $+0.17 \pm 0.19(5)$ & $+0.23 \pm 0.26(7)$ & $+0.12(2)$ \\
\hline $\mathrm{Nd}$ & $+0.22 \pm 0.28(7)$ & - & $+0.26 \pm 0.24(10)$ & $+0.34(2)$ & $+0.26 \pm 0.14(10)$ & $+0.18 \pm 0.20(5)$ & $+0.31 \pm 0.17(4)$ \\
\hline
\end{tabular}

versus excitation potential and Fe I versus reduced equivalent width $\left(\mathrm{W}_{\lambda} / \lambda\right)$ relations. The standard deviation in $\log g$ was set by changing this parameter around the adopted solution until the difference between Fe I and Fe II mean abundance differed by exactly one standard deviation of the $[\mathrm{Fe} / \mathrm{H}]$ mean value. We estimated typical uncertainties in the atmospheric parameters on the order of $\pm 120 \mathrm{~K}, \pm 0.2$ dex and $\pm 0.3 \mathrm{~km} \mathrm{~s}^{-1}$, respectively, for $T_{\text {eff }}, \log g$ and $\xi$.

\subsection{Abundance analysis}

Chemical element abundances of the target stars were determined with the local-thermodynamic-equilibrium (LTE) model-atmosphere techniques. In brief, equivalent widths are calculated by integration through a model atmosphere and compared with the observed equivalent widths. The calculation is repeated, changing the abundance of the element, until a match is achieved. The line-synthesis code MOOG was used to carry out the calculations. The results are given in Table 3, where we list the abundance ratio of element to iron $([\mathrm{X} / \mathrm{Fe}])$, its standard deviation and the number of lines employed for each species (\#). These ratios were based on a solar iron abundance of $\log \epsilon_{\odot}(\mathrm{Fe})=7.52$.

\section{Discussion}

\subsection{HD 87526: a metal-poor star in NGC 3114}

Compared to the metallicity of the other six red giants, HD 87526 (G3) is a metal-poor star. According to González \& Lapasset (2001), HD 87526 is located in the Hertsprung gap, where many binaries (red giant + main sequence star) are encountered in other clusters, although they could not detect any variation based on four radial-velocity measurements.

\subsection{Metallicity and metal distribution}

From the mean of $[\mathrm{Fe} \mathrm{I} / \mathrm{H}]$ values in Table 2 , we obtained a metallicity of $[\mathrm{Fe} / \mathrm{H}]=$ $+0.05 \pm 0.13 \mathrm{dex}$, which corresponds to solar metallicity $(Z=0.02)$. We have excluded 
HD 87526 from this average, since the metallicity we found for this star is very low $([\mathrm{Fe} / \mathrm{H}]=-0.74 \mathrm{dex})$ compared to the other cluster members and we believe it may not be representative of cluster's mean metallicity. Clariá et al. (1989) derived a mean photometric metallicity of $[\mathrm{Fe} / \mathrm{H}]=-0.04$, in good agreement with ours.

Our metallicity is also in accordance with Sestito et al.'s $(2008)$ radial gradient $([\mathrm{Fe} / \mathrm{H}]$ versus galactocentric distance) for open clusters.

\subsection{Masses and ages}

Using our results for $T_{\text {eff }}, \log g$ and $[\mathrm{Fe} / \mathrm{H}]$, and adopting the values of distance modulus, reddening and selective absorption from González \& Lapasset (2001) and the bolometric correction of Alonso et al. (1999; their equation 18), we estimated masses for each of our sample objects. The values that we found vary from 0.84 to $2.71 \mathrm{M}_{\odot}$. The errors, however, are quite large (about 50\%).

Ages were estimated using stellar isochrones from Bertelli et al. (1994) for solar chemical composition $(Z=0.020, Y=0.280)$. We found good agreement with the curve for $\log \tau=8.2\left(\tau=1.6 \times 10^{8} \mathrm{yr}\right)$, in accordance with González \& Lapasset (2001).

\section{Conclusions}

We derived the chemical composition of seven red giants members in the open cluster NGC 3114. We obtained atmospheric parameters as well as the abundances of $\mathrm{O}, \mathrm{Mg}$, $\mathrm{Al}, \mathrm{Si}, \mathrm{Ca}, \mathrm{Ti}, \mathrm{Ni}, \mathrm{Cr}, \mathrm{Sc}, \mathrm{Y}, \mathrm{Zr}, \mathrm{La}, \mathrm{Ce}$ and Nd. We did not find any intrinsic star-to-star scatter in the [element/Fe] ratios for the stars in this cluster.

We derived a mean metallicity of $[\mathrm{Fe} / \mathrm{H}]=0.05 \pm 0.13$ dex, consistent with the solar value. This is also in accordance with the Galactic-disk metallicity distribution for open clusters. Finally, we estimated an age of $\tau=1.6 \times 10^{8}$ yr $(\log \tau=8.2)$ for NGC 3114 .

\section{References}

Alonso, A., Arribas, S., \& Martínez-Roger, C. 1999, A\&ASS, 140, 261

Bertelli, G., Bressan, A., Chiosi, C., Fagotto, F., \& Nasi, E. 1994, A\&SAS, 106, 275

Carraro, G. \& Patat, F. 2001, A\&A, 379, 136

Clariá, J. J., Lapasset, E., \& Minniti, D. 1989, A\&AS, 78, 363

Feinstein, A. 1995, Rev. Mex. A\&BA, 2, 57

González, J. F. \& Lapasset, E. 2001, AJ, 121, 2657

Jankowitz, N. E. \& McCosh, C. J. 1963, MNSSA, 22, 18

Junqueira, S. \& Pereira, C. B., 2001, AJ, 122, 360

Kaufer, A., Stahl, O., Tubbesing, S., Nørregaard, P., Avila, G., Francois, P., Pasquini, L., \& Pizzella, A. 1999, ESO Messenger, 95, 8

Mermilliod, J. C., Mayor, M., \& Udry, S. 2008, A\&A, 485, 303

Paunzen, E., Pintado, O. I., \& Maitzen, H. M. 2003, A\& $A$, 412, 721

Pereira, C. B. 2005, AJ, 129, 2469

Pereira, C. B. \& Roig, F. 2009, AJ, 137, 118

Sagar, R. \& Sharples, R. M. 1991, A\&AS, 88, 47

Schneider, H. \& Weiss, W. W. 1988, A\&AS, 75, 353

Sestito, P., Bragaglia, A., Randich, S., Pallavicini, R., Andrievsky, S. M., \& Korotin, S. A. 2008, $A \mathscr{E} A, 488,943$ 